\title{
El consumo de alcohol en personal administrativo y de servicios de una universidad del Ecuador
}

\author{
Carmita María Bravo Ortiz ${ }^{1}$ \\ Maria Helena Palucci Marziale ${ }^{2}$
}

Se trata de un estudio descriptivo. El objetivo de la investigación fue describir el consumo de alcohol entre los trabajadores en el sector administrativo y de servicios en una universidad en Ecuador y determinar las diferencias en el consumo entre los dos grupos de trabajadores. Participaron 102 trabajadores respondiendo al Cuestionario de Identificación de los Trastornos debidos al Consumo de Alcohol (AUDIT). Los resultados mostraron que el personal de servicios consume más alcohol que el administrativo con un promedio de puntuación total de 7,26 frente a 1,84; presencia de consumo sensato 79,41\%; consumo perjudicial 19,61\%; y, dependencia plena 0,98\%. En el nivel 1 de riesgo de consumo están 76,47\%; en el nivel 2, 18,63\%; en el nivel 3, 3,92\%; y, en nivel 4, 0,98\%. Se concluyó que frente a la identificación del riesgo de consumo encontrado, es necesaria la implantación de un programa de prevención de uso de drogas en la institución estudiada.

Descriptores: Salud Laboral; Consumo de Bebidas Alcohólicas; Alcoholismo; Trabajo.

\footnotetext{
${ }^{1}$ Licenciada en Enfermería, Magíster en Educación Superior. Profesor, Escuela de Enfermería, Universidad Estatal de Guayaquil, Ecuador. E-mail: carmitabravortiz@hotmail.com.

2 Enfermera, Doctor en Enfermería, Profesor Titular, Escola de Enfermagem de Ribeirão Preto, Universidade de São Paulo, Centro Colaborador de la OMS para el Desarrollo de la Investigación en Enfermería, Brasil. E-mail: marziale@eerp.usp.br.
}

Correspondencia:

Maria Helena Palucci Marziale

Universidade de São Paulo. Escola de Enfermagem de Ribeirão Preto

Av. Bandeirantes, 3900

Bairro Monte Alegre

CEP: 14040-902 Ribeirão Preto, SP, Brasil

E-mail: marziale@eerp.usp.br 


\section{O consumo de álcool em pessoal administrativo e de serviço de uma universidade do Equador}

Trata-se de pesquisa descritiva. O objetivo da pesquisa foi descrever o consumo de álcool entre trabalhadores do setor administrativo e de serviços de uma universidade do Equador e verificar diferenças de consumo, entre os dois grupos de trabalhadores. Dos participantes, 102 responderam ao Questionário de Identificação de Transtornos devidos ao Consumo de Álcool (AUDIT). Os resultados mostraram que o pessoal de serviço consume mais álcool que o administrativo, com média de pontuação total de 7,26 e 1,84, respectivamente; presença de consumo sensato entre os funcionários foi de $79,41 \%$, consumo prejudicial de $19,61 \%$ e dependência plena 0,98\%. No nível 1 de risco de consumo estão $76,47 \%$ dos trabalhadores, no nível 2 estão cerca de 18,63\%, no nível 3 cerca de $3,92 \%$ e no nível $4,0,98 \%$ das pessoas. Conclui-se que, frente à identificação de riscos de consumo encontrados, é necessário a implantação de um programa de prevenção ao uso de drogas na instituição estudada.

Descritores: Saúde Ocupacional; Consumo de Bebidas Alcoólicas; Alcoolismo; Trabalho.

\section{Alcohol Consumption in Administrative and Service Personnel in an Ecuadorian University}

The aim of this descriptive study was to characterize the consumption of alcohol among workers in the administrative and service sectors at an Ecuadorian university and to determine differences in consumption between the two groups of workers. The Alcohol Use Disorders Identification Test (AUDIT) was applied to 102 participants. The results showed that the service personnel consumed more alcohol than the administrative personnel with a mean total score of 7.26 against 1.84 . The total prevalence of nonprejudicial consumption was $79.41 \%$, prejudicial consumption $19.61 \%$ and dependency $0.98 \%$. The total scores of $76.47 \%$ of the participants were within risk zone one; $18.63 \%$ risk zone two; $3.92 \%$ risk zone three; $0.98 \%$ risk zone four. In conclusion, due to the identification of hazardous consumption, it is necessary to implement a program of alcohol use prevention in the institution studied.

Descriptors: Occupational Health; Alcohol Drinking; Alcoholism; Work.

\section{Introducción}

El consumo y dependencia de drogas afecta de modo significativo la calidad de vida de las personas; en razón de su magnitud, trascendencia y elevado costo social y económico, se lo considera uno de los más graves problemas de salud pública, con consecuencias psicosociales, como la disminución de la productividad laboral y académica y la violencia familiar(1).

De acuerdo a la ONU(2) la mayoría de los consumidores tiene entre 18 y 35 años, son trabajadores que trasladan su comportamiento a sus centros de trabajo con la consiguiente difusión, originando así problemas graves, como por ejemplo: disminución de la productividad, la calidad y la competitividad, afecta la salud de los trabajadores, aumenta los accidentes, el absentismo, los gastos sociales, y los sanitarios. Así, los productos y servicios ofrecidos son de mala calidad o defectuosos, se crean riesgos de seguridad y salud para el público consumidor, que es quien está pagando los gastos sociales y sanitarios mencionados.

El consumo de alcohol en el trabajo es asociado, en nuestra cultura, a muchos mitos; se piensa que esta sustancia cumple "distintas funciones en la vida diaria de los trabajadores"(3), en relación a distintas condiciones de trabajo; por ejemplo, se bebe alcohol para obtener 
la sensación de energía después de un esfuerzo físico intenso, para relacionarse con clientes y compañeros, para mitigar la sed y el frío, para enfrentar problemas laborales, para integrarse a la cultura de la empresa, entre otros.

La $\operatorname{OIT}^{(3)}$ describe que entre $3 \%$ y $5 \%$ de la población laboral son dependientes del alcohol y $25 \%$ son bebedores de riesgo; en un estudio realizado en los últimos 10 años sobre la prevalencia del consumo de alcohol y accidentes en el trabajo, se encontró que entre $15 \%$ y $30 \%$ de los accidentes mortales ocurridos en el trabajo se deben al consumo de drogas; entre $20 \%$ a $25 \%$ de los accidentes laborales ocurren en personas en estado de intoxicación y los consumidores de alcohol padecen de 2 a 4 veces más accidentes, siendo el ausentismo laboral 2 a 3 veces mayor que el de los demás empleados. En general el porcentaje de trabajadores clasificados como consumidores abusivos de alcohol va desde $40 \%$ hasta $70 \%$.

En Ecuador, cada año se gasta más en el consumo de cerveza, se compraron 16 millones de dólares más en $2003^{(4)}$, siendo éste consumo bajo si comparado con otros países de la región. Pero, ha evolucionado de 18 a 25 litros de consumo por persona al año, variando por regiones, en la región serrana fueron 8 y en la costeña entre 35 y 40 litros por persona. Los estudios epidemiológicos revelan una prevalencia de dependientes adultos que fluctúa alrededor del 7,68\%, lo que añadido a los abusadores, o bebedores problema, llega a constituir el $20 \%$ de la población adulta masculina, siendo este un grupo económicamente activo.

En la segunda encuesta Nacional sobre el consumo de drogas, en 1995, en Ecuador, el Consejo Nacional de control de Sustancias Estupefacientes y Psicotrópicas y el Sistema Ecuatoriano de Vigilancia Integral para la Prevención de Drogas (CONSEP-SEVIP) ${ }^{(5)}$, encontró una prevalencia del consumo de alcohol de 76.4\% en la población que ha consumido alcohol alguna vez en su vida; y que la mitad de la población, (51.2\%), consumió alcohol en el último mes, de éstos últimos el $19.7 \%$ se emborrachó una o más veces; siendo que esta parte de la población podría estar en una situación más problemática que los consumidores ocasionales. También fueron informados excesos ligados al consumo de alcohol, en el intervalo de 12 a 49 años (incidencia importante en la población joven), afectando a tres cuartas partes de esta población.

En el último estudio realizado entre estudiantes de educación media entre los 13 y 19 años de edad por el CONSEP - $2005^{(6)}$ se constató aumento de la prevalencia de vida para el alcohol $(61,6)$ si comparado con el año $1998(53,9)$, y la edad promedio del primer consumo bajó (13,7 años) si comparada con el año 1998 (14.5 años).

En cuanto a la ocupación, las personas que trabajaron parcialmente y bebieron fueron el $85,6 \%$. Al grupo que no tenía ninguna ocupación y que nunca bebió le correspondió el $16.9 \%$ y los desocupados que bebieron fueron el $83.1 \%$. Por categoría de profesión, los mayores niveles de consumo de alcohol se observan en los profesionales, técnicos: en directores y funcionarios públicos, en personal administrativo, en trabajadores agrícolas y no agrícolas y en los conductores de vehículos de transporte; y, los menores niveles de consumo se encuentra entre los comerciantes $y$ trabajadores de servicios.

Por lo tanto el sector laboral está afectado con un alto índice de consumo de alcohol, tabaco y otras drogas, lo que redunda en perjuicio directo en los familiares de los trabajadores, quienes ven postergadas sus apremiantes necesidades de salud, educación, alimentación y vivienda, además del maltrato y el pésimo ambiente afectivo que genera este problema; siendo notorio en ciudades grandes, donde las organizaciones (empresas, instituciones de servicios y otras) que cuentan con un número grande de empleados y en donde existen duras condiciones para desarrollar las actividades laborales sin contar con orientación adecuada, son ambientes propicios para que los empleados sean presa fácil del consumo de alcohol y otras drogas.

Asimismo, según datos no actualizados, hay ausencia de programas de Prevención de Drogas en el sector laboral (90\%) por falta de recursos económicos siendo un problema, que afecta a todos los sectores de la sociedad ecuatoriana, siendo que sus consecuencias repercuten en lo económico, político, cultural, social, laboral y especialmente en la salud, seguridad y soberanía nacional. Además, se mantiene en las empresas, como cultura, la aceptación del alcohol y el cigarrillo, estimulándose este consumo en eventos sociales, culturales y deportivos, en la creencia de este consumo es interesante y la compañía de estas sustancias es satisfactoria.

El problema del abuso del consumo de alcohol en la población laboral, debe ser abordado como en otros países, como Argentina(7), mediante Modelos de Prevención del consumo de drogas y basados en la promoción de la salud, que es el proceso que permite a las personas adquirir mayor control sobre su propia salud y al mismo tiempo mejorarla; estos modelos tienen como finalidad modificar las condiciones de vida, transformar los procesos individuales de toma de 
decisiones favoreciendo la calidad de vida y estimulando

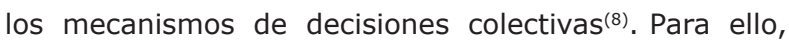
es preciso involucrar al trabajador, a los directivos y/o empresarios, estos dos últimos deben adoptar políticas de prevención de riesgos sobre el consumo de alcohol, abordando problemas asociados al consumo para dar más importancia a la prevención de que al control, y para mejorar los niveles de salud, seguridad y calidad de vida de los trabajadores, que son el motor humano principal de la producción y del desarrollo del país.

Como punto de partida para la modificación de los riesgos asociados al consumo de drogas, lo anterior exige el análisis de los factores protectores y de riesgo del trabajador, que se entretejen en el contexto laboral, considerando los aspectos personales, el contexto social cercano, el entorno social laboral del trabajo y el ambiente familiar.

Los Factores protectores se refieren a las condiciones del ambiente o variables individuales del sujeto que, en constante interacción, contribuyen para reducir las probabilidades de que aparezcan problemas relacionados con la drogas; estos factores apoyan al desarrollo del individuo, sirviendo como amortiguadores o moderadores de los factores de riesgo ${ }^{(7)}$. Por otro lado, los Factores de riesgo son todas aquellas características del individuo y condiciones que exponen a los sujetos o los hacen más vulnerables a caer en el consumo de sustancias psicoactivas, disminuyendo las capacidades para enfrentar las contingencias de la vida de un modo serio y responsable.

\section{Objetivos}

\section{Objetivo General}

Describir el consumo de alcohol entre los trabajadores en el sector administrativo y de servicios en una universidad en Ecuador y determinar las diferencias en el consumo entre los dos grupos de trabajadores.

\section{Objetivos Específicos}

- Identificar la incidencia del abuso de consumo de alcohol en los trabajadores administrativo y de servicios;

- Determinar el nivel de riesgo y tipo de consumo de alcohol por ubicación laboral;

- Relacionar el abuso del consumo de alcohol de acuerdo

a la situación sociodemográfica y tipo de actividad laboral realizada.

\section{Metodología}

Se trata de un estudio descriptivo, trasversal, no experimental con enfoque cuantitativo, realizado en una población total de 134 empleados, la que fue considerada representativa para el estudio. De esa población, 32 no participaron: 9 se recusaron realizando el llenado incompleto del formulario; 10 estaban de vacaciones; 2 con permiso por enfermedad; $y, 11$ no desearon participar. Finalmente la investigación fue realizada con 102 participantes, con categorías de ocupación en personal administrativo (68) y de servicios (34); de los cuales 77 trabajaban en la Escuela de Medicina, 9 en la de Enfermería, 8 en la de Tecnología médica y 8 en Obstetricia de la Facultad de Ciencias Médicas de una Universidad Ecuatoriana, que es una institución laboral de servicio educativo y formación de profesionales de la salud.

Los criterios de inclusión fueron, los trabajadores que hacen parte del cuerpo de personal administrativo y de servicios con nombramiento y contrato de las Escuelas de la Facultad de Ciencias Médicas. Los criterios de exclusión fueron el deseo de no participar o de recusarse a participar.

Los dados fueran recolectados en diciembre de 2007. Para la organización, procesamiento y análisis estadístico de los datos se utilizó el paquete estadístico Statistical Product and Service Solutions SPSS, versión 11.0 para Windows, también se usó el programa Excel, obteniéndose los análisis estadísticos necesarios para la investigación. Estos programas permitieron realizar gráficos estadísticos como diagramas circulares, diagramas de barras y diagramas de caja. El análisis estadístico se dividió en univariado e inferencial; para el análisis inferencial se trabajó con tablas de contingencias, Prueba Chi Cuadrado y análisis de varianza (ANOVA).

\section{Procedimientos éticos}

Para efectuar la presente investigación, se solicitó la aprobación al Comité de Ética en Investigación de la Universidad; también se solicitó la autorización y colaboración a directivos de la Facultad de Ciencias Médicas y respectivas Escuelas. Al grupo participante, para sensibilizarlo, se le explicó el proyecto y se les confirmó el anonimato y absoluta reserva sobre los datos; a continuación se solicitó que firmasen el consentimiento informado libre y esclarecido. 


\section{Instrumentos}

Se utilizó el cuestionario, elaborado con 9 ítems sobre datos sociodemográficos de acuerdo a las variables de estudio: 4 de opinión sobre el consumo de alcohol y 10 reactivos específicos del Cuestionario de Identificación de los Trastornos debidos al Consumo de Alcohol - Tes. de AUDIT(9); la prueba fue auto administrada. El cuestionario fue validado y desarrollado por la OMS (1993) para ser utilizado en la Atención Primaria de la Salud; habiendo sido utilizado en más de 6 países, como método simple de detección del consumo reciente excesivo de alcohol y como apoyo de intervención breve. Este cuestionario es breve, rápido y flexible y es consistente con las definiciones de la CIE-10. El AUDIT determina el abuso del consumo de alcohol, la frecuencia, cantidad, dependencia, daño y consecuencias personales o sociales del bebedor; identifica el nivel de riesgo de consumo de alcohol y a las personas que podrían beneficiarse de la reducción o abandono del consumo.

El rango de las puntuaciones es de 0 a 40; puntuaciones mayores de 20 indican dependencia plena respecto al alcohol; entre 8 y 20 puntos, señalan un consumo perjudicial o de riesgo y una puntuación inferior a 8 no refleja dificultades en el consumo de la sustancia.

El consumo de riesgo es un patrón de consumo de alcohol que aumenta el riesgo de consecuencias adversas para el bebedor o para los demás. El consumo perjudicial conlleva consecuencias para la salud física y mental, así como consecuencias sociales adversas. La dependencia es un conjunto de fenómenos conductuales, cognitivos y fisiológicos que pueden aparecer después del consumo repetido de alcohol.

Los diez reactivos examinan el uso de alcohol durante el último año y evalúan las conductas en relación con el alcohol a través de una escala de tipo Likert. Los reactivos 1 , 2 y 3 evalúan la frecuencia y cantidad típica de alcohol consumido, determinan el consumo de riesgo de alcohol (límite sensato). Los reactivos 4, 5 y 6 , exploran la posibilidad de que exista una dependencia del alcohol; y los reactivos $7,8,9, y 10$, se refieren a la experimentación de consumo dañino de alcohol.

Mediante la puntuación total de AUDIT, se realiza la intervención adecuada, basada en el nivel de riesgo. Hay cuatro niveles de riesgo; el primer nivel o zona I, se refiere al consumo de bajo riesgo o a la abstinencia con puntuaciones de 0 a 7 y la intervención es educación sobre alcohol; El segundo nivel o zona II comprende el consumo de alcohol por encima de las recomendaciones de bajo riesgo indicadas con puntuaciones entre 8 y 15; en este caso se realiza una intervención breve mediante consejo simple y materiales educacionales adecuados. El tercer nivel o zona III con puntuaciones en el rango 16 a 19 corresponde al consumo de riesgo y perjudicial, el que puede ser abordado mediante una combinación de consejo simple, terapia breve y monitorización continuada. Niveles de riesgo con puntuaciones del AUDIT iguales o por encima de 20 , deben ser examinados por un especialista para evaluación diagnostica y tratamiento de la dependencia del alcohol.

\section{Resultados}

Del estudio realizado, más de la mitad de los participantes $(60,4 \%)$ corresponden al género masculino y el $39.6 \%$ al género femenino; la edad más relevante es el grupo de mayores a 46 años. Referente al estado civil, $37 \%$ eran casados, $28 \%$ solteros y $21 \%$ mantenían unión libre; según la escolaridad, el 54\% tenía nivel educativo superior (completa $27,5 \%$ e incompleta 26,5 $\%$ ), y el $27,5 \%$ tenía bachillerato.

En relacion al lugar de trabajo, la mayoría (77,5\%), trabajaban en la escuela de medicina, en menor proporcion en enfermería, obstetricia y tecnología médica. El 67\% desempeñaban cargos administrativos y $33 \%$ de servicios. La variable número de años de trabajo, corresponde a más de 26 años en la institución; la mayoría 94\% trabajaban durante en el día.

La mayoría de los participantes (98\%) opina que el consumo de alcohol es una situación socio cultural y que es un problema que afecta a la salud y la familia, siendo que debe ser atendido; este criterio colocan en evidencia que tienen plena conciencia del problema causado por éste fenómeno.

En el $85 \%$ de los empleados, predomina la cultura del uso de alcohol en momentos en que se reúnen para integrarse con la familia, sea en cumpleaños, navidad, año nuevo, y otros eventos. El tipo de bebida, en orden de preferencia, fue la cerveza, whisky, vino, ron y aguardiente; cerca de las dos terceras partes tienen antecedentes de un familiar muy cercano que consume alcohol.

Se constató la dependencia entre las categorías de las variables Cargo y Cerveza, puesto que la significancia (Sig.) fue menor que 0,05. Los resultados indican que el personal de servicios consume más cerveza que el personal del sector administrativo. 
Tabla 1 - Resultados da prueba de significancia ANOVA

\begin{tabular}{lccccc}
\hline & \multicolumn{5}{c}{ Prueba de Significancia } \\
\cline { 2 - 6 } & SC & G. I. & MC & F & Sig. \\
\hline Tratamiento & 667,46 & 1,00 & 667,46 & 34,41 & 0,00 \\
Error & 1939,84 & 100,00 & 19,40 & & \\
Total & 2607,29 & 101,00 & & & \\
\hline
\end{tabular}

Para comparar los promedios de la puntuación total obtenida al aplicar la prueba de Identificación de Desórdenes en el Uso del Alcohol - AUDIT entre el personal administrativo y de servicio se construyó la tabla ANOVA (Tabla 1); y, basados en la significancia (Sig. $=0,00)$ de la prueba se rechaza la hipótesis nula que indica que los promedios de la puntuación total entre el personal administrativo y de servicios son iguales; $y$, con un $95 \%$ de confianza se establece que existe evidencia estadística para afirmar que los promedios de la puntuación obtenida por parte del personal administrativo y de servicio difieren significativamente entre sí.

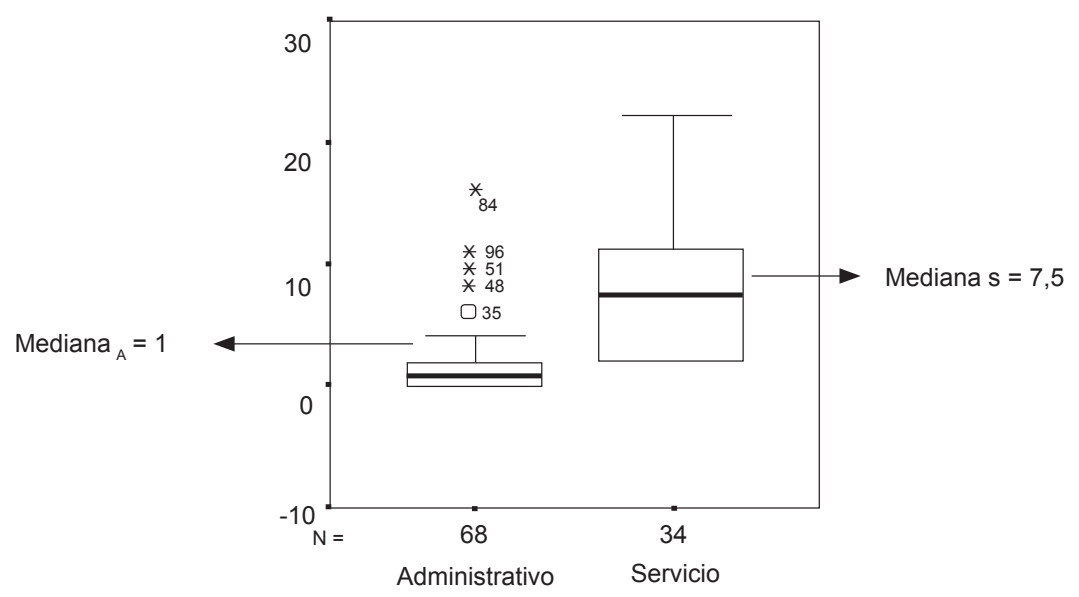

Figura 1 - Diagrama de caja: Puntuación total

El resultado sobre el promedio de la puntuación total obtenido del personal administrativo es 1,84 frente al de personal de servicios 7,26, evidenciándose un promedio de puntuación más alto que el personal administrativo. Además se construyó un diagrama de caja que permita visualizar esta diferencia (Figura 1). El personal de servicios consume más alcohol que el personal administrativo.

Tabla 2 - Frecuencia y porcentaje según el Tipo de Consumo

\begin{tabular}{lccc}
\hline Tipo de Consumo & Frecuencia & Porcentaje & $\begin{array}{c}\text { Porcentaje } \\
\text { Acumulativo }\end{array}$ \\
\hline No perjudicial & 81 & 79,41 & 79,41 \\
Perjudicial & 20 & 19,61 & 99,02 \\
Dependencia plena & 1 & 0,98 & 100,00 \\
Total & 102 & 100 & \\
\hline
\end{tabular}

Fuente: AUDIT

En relación al tipo de consumo sobre la prueba AUDIT (Tabla 2), del total de participantes, 79,41\% presentaron consumo de riesgo (sensato), cerca de la quinta parte $(19,61 \%)$ posee consumo perjudicial, y un pequeñísimo porcentaje $(0,98 \%)$ tiene signos de dependencia plena respecto al alcohol. Por género, el $39,60 \%$ de los hombres y el $39,60 \%$ de las mujeres, muestran consumo sensato; mientras que $19,80 \%$ tienen signos de consumo perjudicial y un mínimo porcentaje, $0,99 \%$ de hombres respectivamente presenta dependencia plena.

Tabla 3 - Frecuencia y porcentaje según cargo

\begin{tabular}{cccc}
\hline \multirow{2}{*}{ Tipo Consumo } & \multicolumn{2}{c}{ Cargo } & Total \\
\cline { 2 - 3 } & Administrativo & Servicios & \\
\hline No perjudicial & 63,00 & 18,00 & 81,00 \\
Frecuencia & 61,76 & 17,65 & 79,41 \\
$\begin{array}{c}\text { \% del Total } \\
\text { Perjudicial }\end{array}$ & & & \\
Frecuencia & 5,00 & 15,00 & 20,00 \\
\% del Total & 4,90 & 14,71 & 19,61 \\
Dependencia plena & & & \\
Frecuencia & & 1,00 & 1,00 \\
$\%$ del Total & & 0,98 & 0,98 \\
Total & & & \\
Frecuencia & 68,00 & 34,00 & 102,00 \\
$\%$ del Total & 66,67 & 33,33 & 100,00 \\
\hline
\end{tabular}

Fuente: AUDIT 
En los resultados que se refieren al tipo de consumo según el Cargo (Tabla 3) el 61,76\% de empleados administrativos presenta consumo no perjudicial, siendo mayor que los empleados de servicios; mientras que el consumo perjudicial alcanza mayores porcentajes $(17,65 \%)$ en el personal de servicios, así como también este grupo tiene signos de dependencia plena $(0,98 \%)$.

En relación al nivel de riesgo del consumo de alcohol, la cuarta parte de los participantes (25\%) alcanza niveles de 2 a 4 de riesgo de consumo. Los resultados indican que en el personal administrativo $y$ en el de servicios existe riesgo de consumo de alcohol.

Tabla 4 - Niveles de Riesgo de consumo de alcohol por género

\begin{tabular}{|c|c|c|c|}
\hline \multirow{2}{*}{ Niveles } & \multicolumn{2}{|c|}{ Género } & \multirow{2}{*}{ Total } \\
\hline & Masculino & Femenino & \\
\hline \multicolumn{4}{|l|}{ Nivel 1} \\
\hline Frecuencia & 37 & 41 & 78 \\
\hline$\%$ de Total & 36,27 & 40,20 & 76,47 \\
\hline \multicolumn{4}{|l|}{ Nivel 2} \\
\hline Frecuencia & 19 & 0 & 19 \\
\hline$\%$ de Total & 18,63 & 0,00 & 18,63 \\
\hline \multicolumn{4}{|l|}{ Nivel 3} \\
\hline Frecuencia & 4 & 0 & 4 \\
\hline$\%$ de Total & 3,92 & 0,00 & 3,92 \\
\hline \multicolumn{4}{|l|}{ Nivel 4} \\
\hline Frecuencia & 1 & 0 & 1 \\
\hline$\%$ de Total & 0,98 & 0,00 & 0,98 \\
\hline \multicolumn{4}{|l|}{ Total } \\
\hline Frecuencia & 61 & 41 & 102 \\
\hline$\%$ de Total & 59,80 & 40,20 & 100,00 \\
\hline
\end{tabular}

En la Tabla 4 se muestra el nivel de riesgo de consumo por género, donde el $36,27 \%$ de los hombres tienen el Nivel 1 de riesgo, mientras que las mujeres, alcanzan el $40,20 \%$ en el mismo nivel. Pero entre los Niveles 2 a 4 de riesgo, existe el 23,53\% de hombres, evidenciándose que hay abuso del consumo de alcohol en el personal de servicios, entre los hombres.

En relación al cargo, el 50,8\% de los empleados administrativos están en el Nivel 1 de riesgo de consumo de alcohol, y el $16,67 \%$ del personal de servicios. Se determina que cerca de la cuarta parte $(23,57 \%)$ del personal de servicios, se encuentra en los Niveles de riesgo 2 y 4 ; en mínima proporción los administrativos $(8,68 \%)$.

\section{Discusión}

La mayoría de participantes (98\%) opina que el consumo del alcohol es una situación sociocultural; además expresan que es un problema que afecta a la salud y la familia y que debe ser atendido sin demora; este criterio expresado por los participantes coloca en evidencia la plena conciencia sobre el problema que causa éste fenómeno. También, entre el $85 \%$ de los participantes predomina la cultura del uso de alcohol en ocasiones especiales donde se reúne la familia, sea cumpleaños, navidad, y otros eventos sociales; además, cerca de las dos terceras partes tienen antecedentes de un familiar muy cercano que consume alcohol.

Esto guarda mucha relación con las normas culturales y el contexto familiar, los que son agentes de socialización, en los que conviven los participantes y podrían estar influenciando el comienzo y mantenimiento de las conductas de bebida; en estas normas se encuentra actitudes generales, valores hacia el alcohol y modelos de conductas cognitivas, entre ellos modelos abusivos de bebida, lo que puede resultar en un alto riesgo en el abuso de alcohol, según lo describe Bandura en su Teoría del aprendizaje cognitivo social(10).

En cuanto al tipo de bebida, en orden de preferencia se encuentran: cerveza, whisky, vino, ron y aguardiente; datos que coinciden con estudios realizados en Cuba, Perú y México(11-13).

Se sustentó estadísticamente que el personal de servicios en relación al personal administrativo consume cerveza con mayor frecuencia y utilizando el promedio de puntuación total obtenida, sobre el consumo de alcohol de acuerdo al cargo, se determinó igualmente que el personal de servicios tiene un promedio de puntuación más alto $(7,26)$ que el personal administrativo $(1,84)$. Los resultados indican que el personal de servicios consume más alcohol que el personal administrativo; estos datos concuerdan con otros estudios realizados en el ámbito laboral en otros países y en sectores laborales donde también ocurre este fenómeno(13-17).

El consumo de riesgo o sensato está presente en la mayoría del total de participantes en iguales porcentajes tanto en hombres como en mujeres y en las ocupaciones de empleados administrativos y de servicios; sin embargo la quinta parte de participantes de género masculino y que actúan en el área de servicios, presenta consumo perjudicial, existiendo un mínimo de este personal que tiene signos de dependencia plena respecto al alcohol.

Estos datos guardan mucha relación con estudios realizados en poblaciones laborales de México y Cuba ${ }^{12}$ 15), donde también se encontraron porcentajes superiores de grupos de trabajadores bebedores que eran del género masculino. Esto explica que el alcoholismo se encuentra con más frecuencia en el género masculino, 
que tiene mayor probabilidad de presentar este tipo de

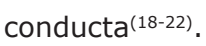

Los resultados alcanzados, establecen que la cuarta parte del personal de servicios de género masculino y en menor proporción el personal administrativo, se encuentran entre los Niveles 2 a 4 de riesgo de consumo. Los resultados indican que en el personal administrativo y de servicio existe riesgo del consumo de alcohol.

En síntesis se determina que un poco más de las tres cuartas partes, de la población estudiada, están en el Nivel 1, siendo este un consumo de riesgo que no presentan dificultades en el consumo de alcohol, debido posiblemente a características individuales de cada persona y a otros factores protectores; así este grupo está muy fortalecido, y los factores ayudan de forma positiva a enfrentar la exposición e influencia del desarrollo del consumo de alcohol.

En consecuencia, estamos frente a un grupo de empleados que demanda Políticas de prevención del consumo de alcohol y atención a la Salud Laboral que sean orientadas a instaurar programas preventivos en adicciones; debiendo contar para ello con Profesionales de Enfermería especializados en la temática, los que tienen como propósito el fortalecimiento del individuo como un ser humano en su integridad, apoyando prácticas de vida saludables y potencializando tratamientos que le permitan recuperar la salud.

\section{Conclusiones}

El estudio permitió conocer que en la población participantes predomina el género masculino y 7 de cada 10 tienen cargo administrativo, sin embargo los hallazgos reportan que hay incidencia de consumo de alcohol entre hombres, del grupo de trabajadores de la universidad, del área de servicios.

Existe variación significativa en los promedios de puntuación total obtenidos al aplicar la prueba AUDIT, determinándose que el personal de servicios consume más alcohol (cerveza) que el personal administrativo.

En relación al tipo de consumo sobre la prueba AUDIT la mayoría presentan consumo de riesgo (sensato) tanto el personal de servicio como administrativo, hombres y mujeres con iguales porcentajes.

Cerca de la quinta parte de participantes hombres con cargo en el personal de servicios, poseen consumo perjudicial y un mínimo porcentaje tiene signos de dependencia plena respecto al alcohol.

Por último, se estableció que la mayoría de los participantes está en el nivel 1 de riesgo de consumo de alcohol; y, la cuarta parte del personal de servicio de sexo masculino y en menor proporción de personal administrativo se encuentran entre los Niveles 2 a 4 de riesgo de consumo. En el Personal administrativo y de servicios existe riesgo de consumo de alcohol.

\section{Recomendaciones}

Los resultados orientan a realizar otros estudios sobre la temática en este ámbito laboral considerando variables propias del trabajo, entre estos los factores personales, los de contexto social cercano, los del entorno social del trabajo y los de ambiente familiar, que servirán como punto de partida para la modificación de factores de riesgo del consumo de alcohol.

La Universidad donde se realizó el estudio, debe dar un salto importante en la construcción y desarrollo de un modelo de prevención del consumo de drogas en el trabajo, con la participación activa de Profesionales de Enfermería, componente fundamental del equipo de salud, conocedor del fenómeno de las drogas, para cumplir su función social en estos espacios laborales, promoviendo estilos de vida saludables, brindando cuidados efectivos de calidad, sea técnica y humana, y participando en la resolución de los problemas derivados de las adicciones.

Para ello se debe involucrar tanto a directivos como a los trabajadores, que respondan a las necesidades, trabajando de manera conjunta e integrada para optimizar este valioso talento humano de la institución, que es el trabajador.

\section{Agradecimientos}

Agradecemos a la Comisión Interamericana para el Control del Abuso de Drogas/CICAD de la Secretaria de Seguridad Multidimensional/SSM de la Organización de los Estados Americanos/OEA, la Secretaria Nacional de Políticas sobre Drogas/SENAD do Gabinete de Seguridad Institucional/Brasil, la Escuela de Enfermería de Ribeirao Preto de la Universidad de Sao Paulo y Centro Colaborador de la Organización Mundial de la Salud para el Desarrollo de la Investigación en Enfermería, la población representada en los estudios de investigación, bien como a las autoridades de las universidades representadas por los participantes del Programa En-Line de Especialización en Investigación sobre el Fenómeno de las Drogas - PREINVEST, periodos 2005, 2006, 2007 y 2008. 


\section{Referencias}

1. Comisión Interamericana para el Control del Abuso de Drogas - CICAD/ Organización de Estados Americanos - OEA (USA). In: Organización Panamericana de la Salud/Organización Mundial de la Salud, (OPS/OMS). La dependencia de las Drogas y su tratamiento. Una experiencia en las Amèricas. Washington D.C.; 2000. p. 8-119.

2. Valencia JE, Pérez GA. Ausentismo laboral y su relación con el consumo de alcohol y otras sustancias psicoactivas. [citado en: 26 nov 2006] [versión en línea]. Disponible en: www.cicad.oas. org/OID/MainPage/Costs/Protocols/Ausentismo.pdf

3. Organización Mundial de la Salud. Informe sobre la salud en el mundo 2002. Reducir los riesgos y promover una vida sana. Ginebra; 2002.

4. Consejo Nacional de control de Sustancias Estupefacientes y Psicotrópicas (EC). Rev Electrónica Prev [CD ROM]. Ecuador: CONSEP; 2004.

5. Consejo Nacional de control de Sustancias Estupefacientes y Psicotrópicas y el Sistema Ecuatoriano de Vigilancia Integral para la Prevención de Droga (EC). Segunda encuesta nacional sobre consumo de drogas. Auspicio Oficina Antinarcóticos. Embajada de los Estados - Unidos (NAS). Ecuador; 1995. p. 41.

6. Consejo Nacional de control de Sustancias Estupefacientes y Psicotrópicas (EC). Segunda Encuesta Nacional Sobre Consumo de Drogas en Estudiantes de enseñanza Media. Naciones Unidas; Oficina contra la Droga y el Delito. Observatorio Ecuatoriano de Drogas, [CD-ROM]. Ecuador; 2005. p. 13.

7. Consejo Nacional para el control de Estupefacientes de Chile (Cl.). Gobierno de Chile. Política de prevención del consumo de drogas en el mundo laboral. Santiago de Chile; 2003.

8. Betancur F. Salud Ocupacional: Un enfoque Humanista. Colombia; McGrawhill; 2001. p. 4.

9. Thomas FB, John CH-B, John BS, Maristela GM. Cuestionario de Identificación de los Transtornos debidos al Consumo de Alcohol (AUDIT): Pautas para su utilización en Atención Primaria. OMS/Departamento de Salud Mental y Dependencia de Sustancias. [citado en: 17 nov 2006] [versión en línea]. Disponible en: http://www.who.int/substance_abuse/activities/ en/AUDITmanualSpanish.pdf

10. Bandura, A. Social foundations of thought \& action: a social cognitive theory. New Jersey: Prentice may; 1986.

11. Magallón TJ, Robazzi ML. Alcohol consumption among industrial workers in Monterrey, Mexico. Rev. Latino-Am. Enfermagem. [serial on the Internet]. 2005 Oct [cited 2007 Feb 25];13(spe): 819-26. Available from: http://www.scielo.br/ scielo.php
12. Cortaza L, Alonso MM. Afecto y consumo de alcohol en trabajadores de la industria petroquímica del sur de Veracruz, México. SMAD Rev Electrónica Salud Mental, Alcohol y Drogas. 2007.[en línea]:1806-6976 Ribeirão Preto [citado en: 12 junio 2007]. Disponible en: rev_smad@eerp.usp.br

13. Guibert W, Cobas S, Reyes R, Reyes Sosa R, Govin Zuaznábar R. Comportamiento ante la ingestión de bebidas alcohólicas. Rev Cubana Med Gen Integr. [en línea]. mar-abr 1999;15(2):10914. [citado 27 julio 2007]. Disponible en: http://scielo.sld.cu/ scielo.php

14. Hernández V, Cabrera C, Blanco JA. Repercusión del Alcoholismo en la vida laboral de un complejo agroindustrial. Rev Med Electrónica 2003; [citado 15 julio 2007]. [en línea]:

Disponible en: www.cpimtz.sld.cu/revista\%20medica/ano\%20 2006/vol5\%202006/tema12.htm

15. Bejarano J, Zamora E, Sánchez AL, Barrantes O. El consumo de alcohol y otras drogas en el Ministerio de Salud de Costa Rica. Rev Costarric Salud Pública;4(7):13-21, dic. 1995.

16. Trucco M, Rebolledo P, González X, Correa A, Bustamante M. Consumo reciente de alcohol y drogas en accidentes del trabajo. Rev Med Chile oct 1998;126(10):1262-7.

17. Caracciolo G B. Consumo de álcool e acidentes do trabalho. J Bras Med. out 1991;61(4):96-8.

18. Becoñas E. Bases científicas de la prevención de las drogodependencias. Delegación del Gobierno para el Plan Nacional sobre Drogas. Madrid (ES); 2002. p. 32-59.

19. Alonso Castillo BA, Marziale MHP, Alonso Castillo MM, Guzmán Facundo FR, Gómez Meza MV. Stressful situations in life, use and abuse of alcohol and drugs by elderly in Monterrey, Mexico. Rev. Latino-Am. Enfermagem. 2008 Aug; 16( no.spe):509-15.

20. Silva J, Ventura CAA, Vargens OMC, Loyola CMD, Eslava ADG, Diaz J, et al. Illicit drug use in seven Latin American countries: critical perspectives of families and familiars. Rev. Latino-Am. Enfermagem. 2009;17(spe):763-9.

21. Gallegos TRM, Brands B, Adlaf E, Giesbrecht N, Simich L, Wright MGM. Aspectos legales del uso de drogas ilícitas en México. Rev. Latino-Am. Enfermagem. 2009;17(spe):783-7.

22. Molina AS, Miasso AI. Benzodiazepine use among employees of a private company. Rev. Latino-Am. Enfermagem. Ago 2008; 16(spe):517-22. 